\title{
Artificial Neural Networks in Drug Design II: Influence of Learning Rate and Momentum Factor on the Predictive Ability $\$$
}

\author{
D. Kaiser ${ }^{1}$, C. Tmej ${ }^{1}$, P.Chiba ${ }^{2}$, K.-J. Schaper ${ }^{3}$, G. Ecker ${ }^{1}$ * \\ 1 Institute of Pharmaceutical Chemistry, University of Vienna, Althanstraße 14, A-1090 Vienna, Austria \\ ${ }^{2}$ Institute of Medical Chemistry, University of Vienna, Währingerstraße 10, A-1090 Vienna, Austria \\ ${ }^{3}$ Medicinal and Pharmaceutical Chemistry, Research Center Borstel, Parkallee 1-40, D-23845 Borstel, Germany
}

\section{Key words}

Modulators of multidrug resistance, propafenone, artificial neural network, P-glycoprotein

\begin{abstract}
A data set of 48 propafenone-type modulators of multidrug resistance was used to investigate the influence of learning rate and momentum factor on the predictive power of artificial neural networks of different architecture. Generally, small learning rates and medium sized momentum factors are preferred. Some of the networks showed higher cross validated $Q^{2}$ values than the corresponding linear model $(0.87$ vs. 0.83$)$. Screening of a 158 compound virtual library identified several new lead compounds with activities in the nanomolar range.
\end{abstract}

\section{Introduction}

Artificial neural networks (ANNs) gain increasing interest in the field of drug design. After a proper learning procedure, ANNs should be able to ,recognize“ basic correlations in a given data set and to predict e.g. physicochemical properties and pharmacological activity. Recently, both Sadowski \& Kubinyi ${ }^{1}$ and Ajay et al. ${ }^{2}$ showed, that ANNs can be trained to predict whether a molecule is drug like or non-drug like. An identical approach was used for the design of combinatorial libraries with CNS activity ${ }^{3}$. The ability for ANNs to deal with non-linear dependencies should favour their use for quantitative structure-activity relationship (QSAR) studies of complex biological phenomena, such as multidrug resistance. Although 
one of the basic underlying mechanisms for development of multiple drug resistance, namely overexpression of drug transport systems such as P-glycoprotein (PGP), is well characterized, the interaction of inhibitors with PGP is not fully understood yet ${ }^{4}$. On a molecular basis, both „unspecific" membrane interaction and "specific" protein interaction seem to take place simultaneously ${ }^{5}$. Systematic QSAR-studies on propafenone-type (1) inhibitors of PGP clearly revealed, that at least for e.g. diphenylalkylamines (2) the observed $\mathrm{EC}_{50}$ values for efflux inhibition may reflect a combination of both interaction modes. ${ }^{6}$ Thus, ANNs should be a versatile tool for QSAR-analysis of modulators of multidrug resistance. Although the use of ANNs usually does not allow to get concrete information on how to modify chemical structures, a well trained ANN may be a versatile tool for screening large virtual libraries to identify new lead compounds. In a preliminary study we were able to demonstrate, that ANNs trained with a combination of Hansch- and Free-Wilson type descriptors as input variables show good predictive ability for PGP-inhibitory activity ${ }^{7}$. The present study focuses on a further optimization of the training conditions.<smiles>CCCNCC(O)COc1ccccc1C(=O)CCc1ccccc1</smiles><smiles>O=C(CCc1ccccc1)c1ccccc1OCC(O)CNCCC(c1ccccc1)c1ccccc1</smiles>

\section{Methods}

\section{Data set}

The data set used consists of 48 propafenone-type MDR-modulators previously described in a QSAR study ${ }^{8}$. Each compound is characterised by 4 physicochemical parameters and 21 indicator variables. On principle, both variations in the vicinity of the nitrogen atom and the phenone moiety were introduced. Therefor, 19 Free-Wilson type indicator variables $\left(\mathrm{X}_{1}-\mathrm{X}_{19}\right)$ were used to describe the different substitution pattern with $\mathrm{X}_{1}-\mathrm{X}_{7}$ denoting variations on the nitrogen atom and $\mathrm{X}_{8}-\mathrm{X}_{19}$ encoding for changes in the phenone moiety. In general, descriptors defined structural differences with respect to the lead molecule propafenone. The indicator variables $I_{m}$ and $I_{p}$ encode for the substitution pattern on the central aromatic ring system. 


\begin{tabular}{|l|l|}
\hline$X_{1}$ & \\
\hline$X_{3}$ & \\
\hline$X_{5}$ & \\
\hline$X_{6}$ & \\
\hline
\end{tabular}

\begin{tabular}{|l|l|}
\hline $\mathrm{X}_{8}$ & (ortho) $\mathrm{CH}(\mathrm{OH}) \mathrm{C}_{2} \mathrm{H}_{4} \mathrm{Ph}$ \\
\hline $\mathrm{X}_{9}$ & (ortho) $\mathrm{CH}\left(\mathrm{OCH}_{3}\right) \mathrm{C}_{2} \mathrm{H}_{4} \mathrm{Ph}$ \\
\hline $\mathrm{X}_{10}$ & (ortho) $\mathrm{COCH}_{3}$ \\
\hline $\mathrm{X}_{11}$ & (ortho) $\mathrm{COC}_{2} \mathrm{H}_{4} \mathrm{Naphth}$ \\
\hline $\mathrm{X}_{12}$ & (ortho) $\mathrm{COC}_{2} \mathrm{H}_{5}$ \\
\hline $\mathrm{X}_{13}$ & (ortho) $\mathrm{COPh}$ \\
\hline $\mathrm{X}_{14}$ & (ortho $\rightarrow$ para) $\mathrm{COC}_{2} \mathrm{H}_{4} \mathrm{Ph}$ \\
\hline $\mathrm{X}_{15}$ & 5-OH \\
\hline $\mathrm{X}_{16}$ & 5-OCH $\mathrm{Ph}$ \\
\hline $\mathrm{X}_{17}$ & (ortho $\rightarrow$ para) $\mathrm{COCH}_{3}$ \\
\hline $\mathrm{X}_{18}$ & (ortho $\rightarrow$ meta) $\mathrm{COC}_{2} \mathrm{H}_{4} \mathrm{Ph}$ \\
\hline $\mathrm{X}_{19}$ & (ortho $\rightarrow$ meta) $\mathrm{COCH}_{3}$ \\
\hline
\end{tabular}

Table 1: Descriptors $\mathrm{X}_{1}-\mathrm{X}_{19}$

Table 2: Encoding of compounds GP 01 (propafenone) - GP 386<smiles>[Y]CC(O)COc1ccccc1[Y6]</smiles>

\begin{tabular}{|l|l|l|l|l|l|l|l|l|}
\hline & - & $\mathrm{X}_{1}$ & $\mathrm{X}_{2}$ & $\mathrm{X}_{3}$ & $\mathrm{X}_{4}$ & $\mathrm{X}_{5}$ & $\mathrm{X}_{6}$ & $\mathrm{X}_{7}$ \\
\hline & Gp 01 & Gp 05 & Gp 31 & Gp 09 & Gp 57 & Gp 29 & Gp 62 & Gp 238 \\
\hline $\mathrm{X}_{8}$ & & Gp 88 & Gp 155 & Gp 163 & Gp 226 & & & \\
\hline $\mathrm{X}_{9}$ & & Gp 90 & Gp 156 & Gp 164 & Gp 227 & & & Gp 220 \\
\hline$X_{10}$ & & & Gp 45 & & & & Gp 323 & \\
\hline$X_{11}$ & & Gp 180 & & Gp 376 & Gp 374 & & Gp 382 & Gp 184 \\
\hline$X_{12}$ & & Gp 12 & & & Gp 46 & & Gp 381 & \\
\hline$X_{13}$ & & & Gp 319 & & & & Gp 317 & \\
\hline$X_{14}$ & & Gp 73 & Gp 134 & Gp 159 & Gp 149 & & & \\
\hline$X_{15}$ & Gp 129 & Gp 233 & & & & Gp 254 & & \\
\hline$X_{16}$ & Gp 231 & Gp 232 & & Gp 246 & & Gp 253 & & \\
\hline$X_{17}$ & & Gp 389 & Gp 390 & & Gp 391 & & & \\
\hline$X_{18}$ & & Gp 135 & Gp 157 & & & & & \\
\hline$X_{19}$ & & Gp 385 & Gp 386 & & Gp 384 & & & \\
\hline
\end{tabular}


For description of physicochemical properties both calculated $\log P$ and molar refraction (MR) were used. Corresponding values were calculated using the software package MOLGEN (for further details see ref. 8). Both parameters were shown to be highly predictive for PGPinhibitory activity. In order to characterize the influence of lipophilicity in more detail, the partial $\log \mathrm{P}$ values of the substituent on the nitrogen atom $\left(\log \mathrm{P}_{\mathrm{N}}\right)$ and on the central aromatic ring moiety $\left(\log \mathrm{P}_{\mathrm{ar}}\right)$ were also calculated.

\section{PGP-inhibitory activity}

For measurement of pharmacological activity the daunomycin efflux assay was used. ${ }^{9}$ Briefly, PGP expressing CCRF-CEM vcr1000 T-lymphoblast cells ${ }^{9}$ were incubated with medium containing $3 \mu \mathrm{mol} / 1$ daunomycin to reach a steady state of accumulation. Extracellular fluorochrome was removed and cells were resuspended in medium containing either no modulator or chemosensitizer at various concentrations. Generally, 8 serial dilutions were tested for each modulator. After 1, 2, 3 and 4 min aliquots of the incubation mixture were drawn and measured on a Becton Dickinson FACScalibur flow cytometer as described. ${ }^{9}$ Parental CCRF-CEM cells were used to compensate for simple membrane diffusion, which was less than $3 \%$ of the efflux rates observed in resistant cells. Dose response curves were fitted to the data points using non-linear least squares and $\mathrm{EC}_{50}$ values were calculated as described. ${ }^{8}$

\section{Artificial neural networks}

Generally, three layer feed forward networks of different architecture were used. All networks were trained using the backpropagation algorithm and a sigmoid transfer function in the hidden layer and output layer. Thus, in addition to the number of hidden layer units the learning rate $(\mathrm{LR})^{10,11,12}$ and the momentum factor $(\mathrm{MF})^{10}$ were identified as the main factors influencing network performance. In preliminary studies the following configurations were determined as being optimal: 25:5:1 (all input variables were used), 19:5:1 (only Free-Wilson type indicator variables $\mathrm{X}_{1}-\mathrm{X}_{19}$ were used) and 7:4:1 (only those descriptors which remained in the final equation of a multiple linear regression analysis were used: $M R, I p, X_{2}, X_{4}, X_{5}$, $\left.\mathrm{X}_{7}, \mathrm{X}_{8}\right)$

For examination of the best training parameters the software package NEUROMAT-NT (K.J. Schaper, Borstel) was modified to enable automatic leave one out cross validation. Additionally, this software package offers the unique feature of a forgetting algorithm. ${ }^{13}$ After a given number of training-epochs all weights are reduced by a small amount (i.e. partial 
forgetting of previously extracted information). Those weights which are important for a proper performance of the network will recover to their original level during consecutive training cycles. Those which are not necessarily involved in description of PGP-inhibitory activity will stay at the new (lower) level. After several thousand cycles, unimportant weights will change to about zero and thus allow to identify neurones which were not important for the description of pharmacological activity.

Although due to the general flexibility of artificial neural networks scaling of variables is not required, a minimum/maximum scaling procedure was applied to transform all variables into a uniform value range. An additional factor was applied to set the maximum to 0.9 and the minimum to 0.1 . This enables the network to gain greater flexibility for prediction of output values beyond the values used in the training procedure.

The momentum factor was varied from 0.01 to $0.8(0.01,0.05,0.1,0.2,0.4,0.8)$ and the learning rate from 0.01 to $0.2(0.01,0.05,0.1,0.2)$. Learning rates higher than 0.2 led to oscillations of the network during training. The number of epochs $(\max 15000)$ and the overall error (max. 0.1) were used as stopping conditions. Each possible combination of MF and LR was used, whereby training of the respective ANNs was performed both with and without forgetting. Each training run started with new randomly selected weights. In summary, 48 different training conditions had to be applied for each network configuration. For each of these 144 networks a leave-one-out cross validation was performed to check the predictive ability.

\section{Results}

\section{Predictive Power}

For the given data set, the predictive power generally is satisfactory with $Q^{2}$-values in between $0.45-0.87$ (Table 3). With the exception of the 25:5:1 network, no statistically significant differences in the mean performance of training with and without forgetting could be observed (t-test).

\begin{tabular}{|c|c|c|c|c|}
\hline & \multicolumn{2}{|c|}{ With forgetting } & \multicolumn{2}{c|}{ Without forgetting } \\
\hline & $\mathrm{R}$ & $\mathrm{Q}_{\mathrm{cv}}^{2}$ & $\mathrm{R}$ & $\mathrm{Q}_{\mathrm{cv}}^{2}$ \\
\hline $25: 5: 1$ & $0.77-0.91$ & $0.52-0.83$ & $0.74-0.88$ & $0.45-0.87$ \\
\hline $19: 5: 1$ & $0.79-0.90$ & $0.54-0.80$ & $0.80-0.91$ & $0.57-0.82$ \\
\hline $7: 4: 1$ & $0.85-0.92$ & $0.67-0.85$ & $0.83-0.91$ & $0.63-0.82$ \\
\hline
\end{tabular}

Table 3: Statistical parameters for predictive power of different networks 


\section{Training conditions}

Figures $1 \mathrm{a}$ and $1 \mathrm{~b}$ show the influence of momentum factor and learning rate on the predictive power (25:5:1). When using NEUROMAT NT small learning rates and medium sized momentum factors are preferred. Learning rates higher than 0.2 lead to oscillation of the network. Nevertheless, this seems to be a specific disadvantage of the training algorithm implemented in NEUROMAT NT. Best cross validated $Q^{2}$ values were obtained with the 25:5:1 network without forgetting, a LR of 0.05 and a MF of 0.1 . This network showed higher predictive ability than the corresponding linear model $\left(Q^{2}{ }_{\mathrm{cv}}=0.83\right)$. Nevertheless, applying a fit procedure to the data points (STATISTICA), optimal MF values seem to be in the range of 0.5 , which is in accordance with values reported in ref. 10 (Figures 1a, 1b).

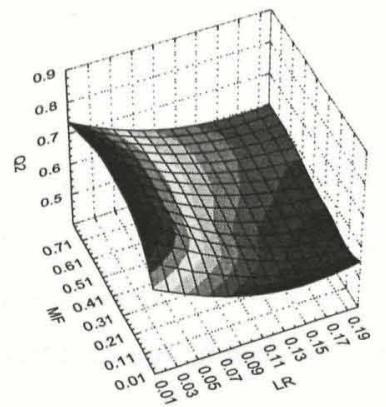

Figure 1a: Influence of learning rate (LR) and momentum factor (MF) on the predictive power (without forgetting)

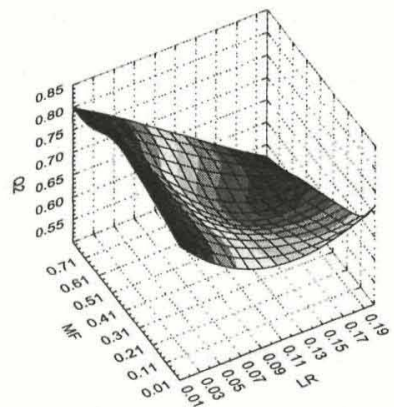

Figure $1 \mathrm{~b}$ : Influence of learning rate (LR) and momentum factor (MF) on the predictive power (with forgetting)

\section{Screening of a small virtual library}

Theoretically, a total of 206 substances, of which 48 are already synthesized and pharmacologically tested, can be described by use of the proposed 21 indicator input variables,. Thus, we generated a virtual library consisting of the remaining 158 compounds. PGP-inhibitory activity of the virtual compounds was predicted using those 3 networks (25:5:1, 19:5:1, 7:4:1), which gave highest $Q^{2}$ values in the leave one out cross validation runs. Due to the fact, that the sigmoid transfer function in the output neurone gives output values in the range of $0-1$, scaling and descaling of the output data is very important. If the scaling routine applied during the training process (usually minimum-maximum scaling) does not allow enough flexibility on both sides of the activity range, the calculated output data will follow the sigmoid transfer function of the output neurone (compare Figures $2 a$ and $2 b$, where activities predicted by ANNs with restricted and relatively unrestricted scaling ranges are 
plotted against activities predicted on basis of a MLR equation). Almost identical results were obtained with all 3 networks with respect to the chemical structure of those compounds predicted as highly active or as almost inactive (Table 4). Generally, 5-benzyloxy-analogues (DK072, DK100) with a 4-phenyl-4-hydroxy-piperidino substituent were predicted as highly active. On the other hand, 5-hydroxy-analogues with a morpholino substituent (DK081, DK151) were predicted as almost inactive (Figure 3).

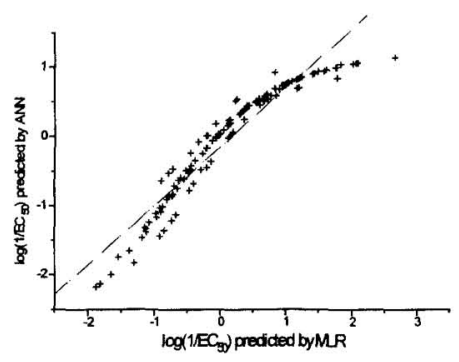

Figure 2a: Scaling for $\min / \max = \pm 1.0$

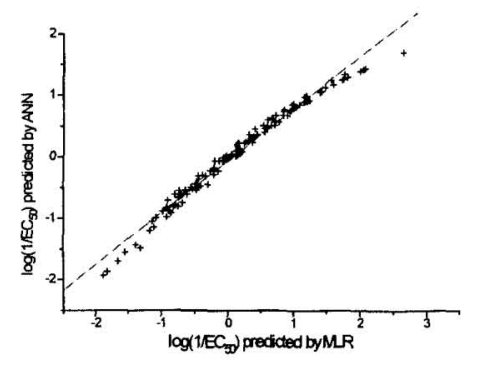

Figure $2 \mathrm{~b}$ : Scaling for $\min / \max = \pm 5.0$

\begin{tabular}{|l|c|c|c|}
\hline & \multicolumn{3}{|c|}{ EC $_{50}$ predicted $(\mu \mathrm{M})$} \\
\hline & $25: 5: 1$ & $19: 5: 1$ & $7: 4: 1$ \\
\hline DK100 & 0.036 & 0.023 & 0.019 \\
\hline DK072 & 0.040 & 0.027 & 0.037 \\
\hline DK081 & 888.6 & 251.1 & 73.9 \\
\hline DK151 & 887.0 & 406.3 & 73.9 \\
\hline
\end{tabular}<smiles>CC(=O)c1cc(O)ccc1OCC(O)CN1CCOCC1</smiles>

DK081<smiles>CC(=O)c1cc(OCC(O)CN2CCOCC2)ccc1O</smiles>

Table 4: predicted $\mathrm{EC}_{50}$ values for selected compounds of the virtual library<smiles>COC(CCC1=CC=CCC1)c1cc(OCc2ccccc2)ccc1OCC(O)CN1CCC(O)(c2ccccc2)CC1</smiles>

Figure 3: Chemical structures of compounds DK072, DK081, DK100 and DK151 
This demonstrates, that indeed some of the main predictive factors for MDR-modulating activity, such as high lipophilicity or the presence of a 4-hydroxy-4-phenyl-piperidyl substituent, were identified and used for prediction of the activity of new compounds. Nevertheless, as shown previously, ${ }^{14}$ in case of a 5-benzyloxy substitution, $\mathrm{EC}_{50}$ values seem to level at $1 \mu \mathrm{M}$, independent of the corresponding $\log \mathrm{P}$ values. Thus, synthesis and pharmacological testing of a range of compounds with different predicted activity will show the validity of concept of virtual library screening using artificial neural networks as a tool for the design of new, highly active compounds.

\section{Acknowledgement}

This work was supported by the "Jubiläumsfond der Stadt Wien für die Akademie der Wissenschaften" Project J/25/99.

\section{References}

1 Sadowski J., Kubinyi H. (1998), J. Med. Chem. 41: 3325

$2 \quad$ Ajay A., Walters W.P., Murcko M.A. (1998), J. Med. Chem. 41: 3314

$3 \quad$ Ajay A., Bemis G.W., Murcko M.A. (1999), J. Med. Chem. $42: 4942$

4 Ambudkar S.V., Dey S., Hrycyna C.A., Ramachandra M., Pastan I., Gottesman M.M. (1999), Annu. Rev. Pharmacol. Toxicol. 39: 361

$5 \quad$ Seydel J.K., Coats E.A., Cordes H.P., Wiese M. (1994), Arch. Pharm. 327: 601

6 Ecker G., Tmej C., Richter E., Hitzler M., Chiba P., Seydel J.K., Fleischhacker W. (1998), Sci. Pharm. 66: S33

7 Tmej C., Chiba P., Schaper K.-J., Ecker G., Fleischhacker W. (1999), in Kaspers et al., Drug Resistance in Leukemia and Lymphoma III, Kluwer Academic/Plenum Publisher, New York, pp 95-105

8 Tmej C., Chiba P., Huber M., Richter E., Hitzler M., Schaper K.-J., Ecker G. (1998), Arch. Pharm. Pharm. Med. Chem. 331: 233

9 Chiba P., Ecker G., Schmid D., Drach J., Tell B., Goldenberg S., Gekeler V. (1996), Mol. Pharmacol. 49: 1122

10 Tollenaere T. (1990), Neural Networks 3: 561

11 Silvia F. M., Almeida L. B. (1990), in: Almeida L. B., Wellekens C. J. (eds.), Neural Networks, Lecture Notes in Computer Sciences, Springer, Berlin 412: 110

12 Weir M. K. (1991), Neural Networks 4: 371

13 Aoyama T., Ichikawa H. (1991), Chem. Pharm. Bull. 39: 1222

14 Chiba P., Tell B., Jäger W., Spatzenegger M., Richter E., Hitzler M., Ecker G. (1997), Arch. Pharm. Pharm. Med. Chem. 330: 343 